\title{
Methodology for Leakage Isolation using Pressure Sensitivity Analysis in Water Distribution Networks
}

\author{
Ramon Pérez*, Vicenç Puig ${ }^{\#, *}$, Josep Pascual*, Joseba Quevedo*, Edson Landeros ${ }^{0}$, Antonio Peralta ${ }^{+}$ \\ *Advanced Control Systems Group (SAC) \\ Universitat Politècnica de Catalunya (UPC) \\ Rambla Sant Nebridi, 10, 08222 Terrassa, Spain \\ e-mail : ramon.perez@upc.edu \\ fax: +349373928 \\ ${ }^{\#} I R I$ Institut de Robòtica i Informàtica Industrial (CSIC-UPC) \\ ${ }^{\circ}$ CETAQUA Water Technological Center \\ ${ }^{+}$AGBAR Barcelona Water Company
}

\begin{abstract}
Leaks are present to some extent in all water-distribution systems. This paper proposes a leakage localisation method based on the pressure measurements and pressure sensitivity analysis of nodes in a network. The sensitivity analysis using analytical tools is not a trivial job in a real network because the huge non-explicit non-linear systems of equations that describe its dynamics. Simulations of the network in presence and absence of leakage may provide an approximation of this sensitivity. This matrix is binarised using a threshold independent of the node. The binary matrix is assumed as a signature matrix for leakages. However, there is a trade-off between the resolution of the leakage isolation procedure and the number of available pressure sensors. In order to maximise the isolability with a reasonable number of sensors, an optimal sensor placement methodology, based on genetic algorithms, is also proposed. These methodologies have been developed for Barcelona Network using PICCOLO simulator. The sensor placement and the leakage detection and localization methodologies are applied to several district management areas (DMA) in simulation and in reality.
\end{abstract}

Keywords: Pressure Sensitivity, Leakage Localisation, Sensor Placement. 


\section{INTRODUCTION}

Water loss in distribution system networks is an issue of great concern for water utilities, strongly linked with operational costs and water resources savings. Continuous improvements in water loss management are being applied and new technologies are developed to achieve higher levels of efficiency. Usually a leakage detection method in a DMA (District Metered Area) starts by analyzing input flow data, such as minimum night flows and consumer metering data (Lambert, 1994) (MacDonald, 2005). Once the water distribution district is identified to have a leakage, various techniques are used to locate the leakage for pipe replacement or repair. Methods for locating leaks range from ground-penetrating radar to acoustic listening devices or physical inspection (Farley, 2003)(Colombo, 2009). Some of these techniques require isolating and shutting down part of the system. The whole process could take weeks or months with a significant volume of water wasted. Techniques based on locating leaks from pressure monitoring devices allow a more effective and less costly search in situ.

This paper presents a model based methodology to detect and localize leaks. It has been developed within a project carried out by Aguas Barcelona, Water Technological Centre CETaqua, and the Technical University of Catalonia (UPC). The objective of this project is to develop and apply an efficient system to detect and locate leaks in a water distribution network. It integrates methods and technologies available and in use by water companies, including DMA and flow/pressure sensor data, in conjunction with mathematical hydraulic models. The method is based on the analysis of pressure variations produced by a leakage in the water distribution network (Pudar, 1992). This technique differs from others in the literature, such as the reflection method (LRM) or the inverse transient analysis (ITA), since it is not based on the transient analysis of pressure waves (Ferrante, 2003a; 2003b)(Misiunas, 2005)(Verde, 2007). Alternatively, the leakage detection procedure is performed by comparing real pressure and flow data with their estimation using the simulation of the mathematical network model and pressure sensitivity analysis of nodes in a network when a leak is present in a node. The analytical calculation of sensitivity is not a trivial job in a real network because the huge non-explicit non-linear systems of equations that describe its dynamics. Simulation of the network in presence and absence of leakage provides an approximation of this sensitivity. The approximation is used to generate a sensitivity matrix that is binarised using a threshold independent of the node. In order to successfully apply this methodology, the characterization of district metered areas and consumers, considered a critical issue for a correct model calibration, should be also addressed but is not described in this paper (see, f.e. (Pérez, 2009a) for further details). The paper also proposes a methodology to place pressure sensors within a DMA that optimizes leakage detection using a minimum number of sensors based on the approach proposed in (Pérez, 2009b). Finally, the leakage detection methodology proposed will be tested with sensors installed in three DMA's used as case studies. 
Section 2 reviews water distribution network modelling and presents the case study used to illustrate the proposed methodologies. Model based fault detection and isolation techniques described in Section 3 are used for the leakage detection and location. Section 4 presents how the leak signature matrix is obtained from the pressure sensitivity matrix. Since the sensor placement is a critical issue for maximising discriminability, an algorithm is presented in Section 5. The signature matrix is generated for the set of sensors selected. This matrix has to be compared with the signature obtained comparing the model and the real measurements. From this comparison, the leakage is located in a set of possible nodes. This methodology is presented in Section 6 and is illustrated in simulation using the proposed case study while Section 7 presents the results obtained in several real scenarios. Finally, Section 8 summarizes the conclusions.

\section{WATER DISTRIBUTION SYSTEMS: PLAÇA DEL DIAMANT CASE STUDY}

A water distribution system consists of three major components: pumps, distribution storage, and distribution piping network. Most systems require pumps to supply lift to overcome differences in elevation, and energy losses caused by friction. Pipes may contain flow-control devices, such as regulating or pressure-reducing valves (Brdys and Ulanicki, 1994). The purpose of a distribution system is to supply the system's users with the amount of water demanded, under adequate pressure for various loading conditions. A loading condition is a spatial pattern of demands that defines the users' flow requirements.

\subsection{Mathematical modelling}

The governing laws for flow in pipe systems under steady conditions are conservation of mass and energy. The law of conservation of mass states that the rate of storage in a system is equal to the difference between the inflow to and outflow from the system. In pressurized water distribution networks, no storage can occur within the pipe network, although tank storage may change over time. Therefore, in a pipe, or a junction node, the inflow and outflow must balance. For a junction node,

$$
\sum q_{\text {in }}-\sum q_{\text {out }}=q_{\text {ext }}
$$

where $q_{\text {in }}$ and $q_{\text {out }}$ are the pipe flow rates into and out of the node and $q_{\text {ext }}$ is the external demand or supply. Conservation of energy states that the difference in energy between two points is equal to the energy added to the flow in components between these points minus the frictional and minor losses. An energy balance can be written for paths between the two end points of a 
single pipe, between two fixed graded nodes (a node for which the total energy is known, such as a tank) through a series of pipes, valves, and pumps, or around a loop that begins and ends at the same point. In a general form for any path,

$$
\sum_{i \in J_{p}} h_{P, j}-\sum_{i \in I_{p}} h_{L, i}=\Delta E
$$

where: $h_{L, i}$ is the headloss across component $i$ along the path, $h_{P, j}$ is the head added by pump $j$, and $\Delta E$ is the difference in energy between the end points of the path. The primary network component is a pipe. The relationship between pipe flow $(q)$ and energy loss caused by friction $\left(h_{L}\right)$ in individual pipes can be represented by a number of equations, including the DarcyWeisbach and Hazen-Williams equations. The general relationship is of the form

$$
h_{L}=K q^{r}
$$

where $K$ is a pipe coefficient that depends on the pipe's diameter, length, and material and $r$ is an exponent in the range of 2.

\subsection{Plaça del Diamant DMA Case Study}

The case study used to illustrate the leak methodology presented in this paper is based on Plaça del Diamant DMA at the Barcelona Water Network (see Figure 1). This DMA is used for illustrating the methodology. Its model contains 1600 nodes and 41.153m of pipes. This DMA is simulated using PICCOLO software. Demands are assumed to occur in the nodes. In this paper, it will also be assumed that leaks occur at the nodes. Then, under such assumption, leaks can be seen as additional demands but with unknown location and quantity.

Simulated leaks introduced in the network are of $1 \mathrm{l} / \mathrm{s}$, more or less $3 \%$ of the total demand of the sector (in the night time). The demand distribution all over the network is the most variable parameter of the model. Some uncertainty in the demand has also been included in order to test the robustness of the method.

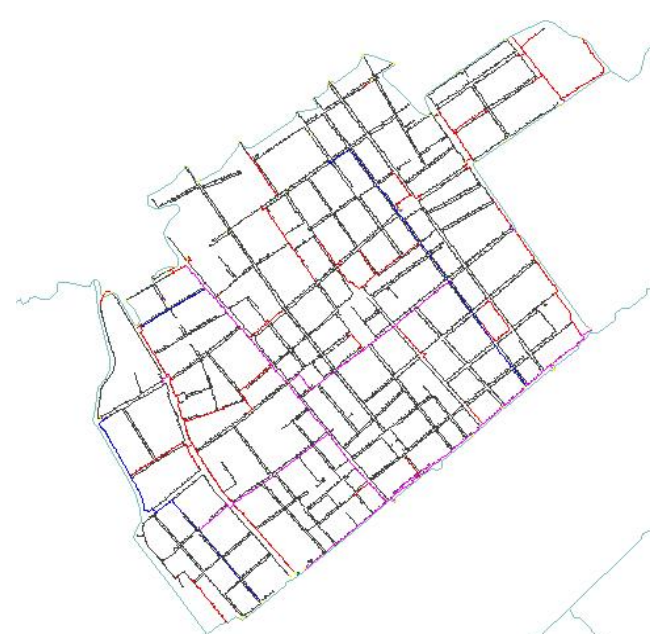

Fig. 1: Case study network: Plaça del Diamant 


\section{LEAKAGE DETECTION AND ISOLATION METHODOLOGY FOUNDATIONS}

The methodology of leakage localisation proposed in this paper is mainly based on standard theory of model-based diagnosis described for example in (Gertler, 1998) that has already been applied to water networks to detect faults in flow meters (Ragot, 2006). Model based diagnosis can be divided in two subtasks: fault detection and fault isolation. The principle of model-based fault detection is to check the consistency of observed behaviour while fault isolation tries to isolate the component that is in fault. The consistency check is based on computing residuals, $\boldsymbol{r}(k)$, obtained from measured input signals $\boldsymbol{u}(k)$ and outputs $\boldsymbol{y}(k)$ using the sensors installed in the monitored system and the analytical relationship which are obtained by system modelling:

$$
\boldsymbol{r}(k)=\boldsymbol{\Psi}(\boldsymbol{y}(k), \boldsymbol{u}(k))
$$

where $\boldsymbol{\Psi}$ is the residuals generator function that depends on the type of detection strategy used (parity equation (Gertler, 1998) or observer (Chen and Patton, 1999)). At each time instance, $k$, the residual is compared with a threshold value (zero in ideal case or almost zero in real case). The threshold value is typically determined using statistical or set-based methods that take into account the effect of noise and model uncertainty (Blanke, 2006). When a residual is bigger than the threshold, it is determined that there is a fault in the system; otherwise, it is considered that the system is working properly. In practice, because of input and output noise, nuisance inputs and modelling errors affecting to the considered model, robust residuals generators must be used. The robustness of a fault detection system means that it must be only sensitive to faults, even in the presence of model-reality differences (Chen and Patton, 1999).

Robustness can be achieved at residual generation (active) or evaluation phase (passive). Most of the passive robust residual evaluation methods are based on an adaptive threshold changing in time according to the plant input signal and taking into account model uncertainty either in the time or frequency domain. In this paper, a passive method in time domain has been proposed for robust fault detection, where the detection threshold has been obtained using the method described in Section 3.

Robust residual evaluation allows obtaining a set of observed fault signatures $\phi(k)=\left[\phi_{1}(k), \phi_{2}(k), \cdots, \phi_{n_{\phi}}(k)\right]$, where each indicator of fault is obtained as follows: 


$$
\phi_{i}(k)= \begin{cases}0 & \text { if }\left|r_{i}(k)\right| \leq \tau_{i}(k) \\ 1 & \text { if }\left|r_{i}(k)\right|>\tau_{i}(k)\end{cases}
$$

where $\tau_{i}$ is the threshold associated to the residual $r_{i}(k)$.

Fault isolation involves identifying the faults affecting the system. It is carried out on the basis of observed fault signatures, $\phi$, generated by the detection module and its relation with all the considered faults, $\boldsymbol{f}(k)=\left\{f(k)_{1}, f_{2}(k), \cdots, f_{n_{f}}(k)\right\}$ that are compared with theoretical signature matrix FSM (Gertler, 1998). One element of this matrix $F S M_{i j}$ will be equal to one, if a fault $f_{j}(k)$ is affected by the residual $r_{i}(k)$, in this case the value of the fault indicator $\phi_{i}(k)$ must be equal to one when the fault appears in the monitored system. Otherwise, the element $F S M_{i j}$ will be zero. A given fault $f_{i}(k)$ is proposed as a fault candidate when the observed fault signature matches with its theoretical fault signature.

\section{LEAKAGE SENSITIVITY ANALYSIS}

The theoretical signature matrix needed to apply the isolation method presented in previous section can be obtained from a leakage sensitivity analysis. This analysis evaluates the effect of a leakage on the pressure in a node. If this process is repeated for each node and possible leak, the sensitivity matrix (Pudar, 1992) is obtained as follows:

$$
S=\left(\begin{array}{ccc}
\frac{\partial p_{1}}{\partial f_{1}} & \cdots & \frac{\partial p_{1}}{\partial f_{n}} \\
\cdot & \cdots & \cdot \\
\frac{\partial p_{n}}{\partial f_{1}} & \cdots & \frac{\partial p_{n}}{\partial f_{n}}
\end{array}\right)
$$

where each element $s_{i j}$ measures the effect of leak $f_{j}$ in the pressure of node $p_{i}$. It is extremely difficult to calculate $S$ analytically in a real network because the model is based on a huge set of implicit non-linear equations as described in Section 2. This work proposes instead generating the sensitivity matrix by simulation using increments of pressure and maintaining constant the leakage flow. It has been verified that the analytical and the simulated sensitivity converge for small leakages. The sensitivity matrix depends on the working point that is, on the demand and boundary conditions (Vento, 2009).

In Figure 2, the sensitivity matrix for the case study network of Figure 1 is shown graphically. It has been ploted for 15 nodes distributed homogenously in the DMA as illustration. 


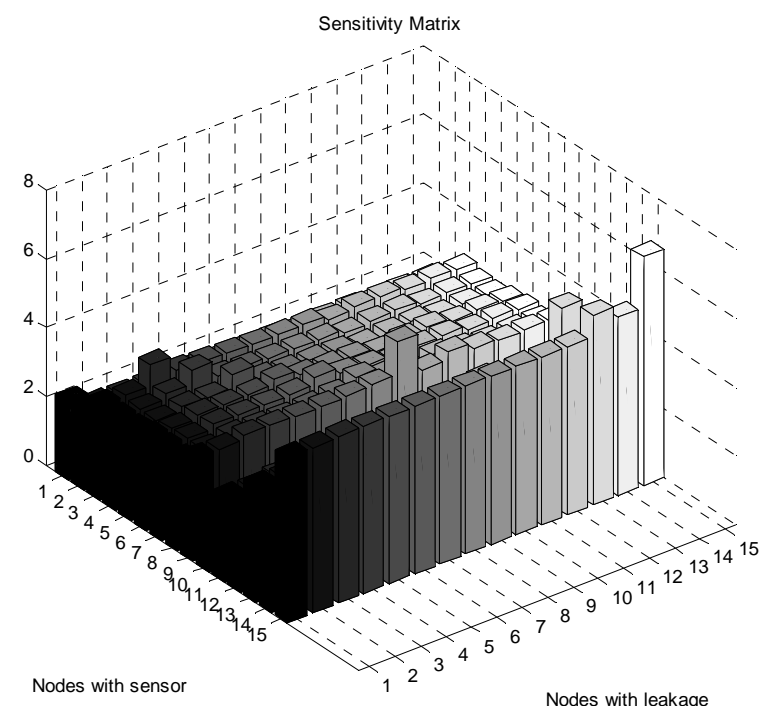

Fig: 2. Sensitivity matrix

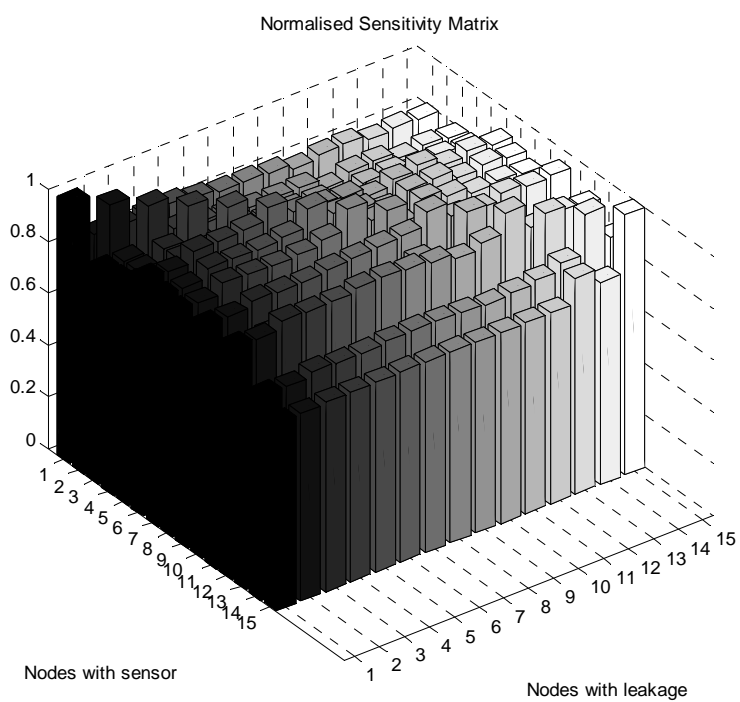

Fig. 3: Normalised sensitivity matrix

Some sensors are much more sensitive to all leakages than others. Thus, a normalisation of sensitivity is needed so that the information provided by any node is comparable. Each row is divided by the maximum value of this row that corresponds to the leakage most important for that node) leading to the normalised sensitivity matrix:

$$
\bar{S}=\left(\begin{array}{ccc}
\frac{s_{11}}{\sigma_{1}} & \ldots & \frac{s_{1 n}}{\sigma_{1}} \\
\cdot & \ldots & \cdot \\
\frac{s_{n 1}}{\sigma_{n}} & \ldots & \frac{s_{n n}}{\sigma_{n}}
\end{array}\right)
$$

where: $\sigma_{i}=\max \left\{s_{i 1}, \cdots, s_{i n}\right\} \quad i=1, \cdots, n$. This matrix is shown in Figure 3 for the considered example. It shows how the most relevant leak is the one on the node itself, the maximum normalised sensitivity is on the diagonal.

Finally, from the normalised sensitivity matrix (7), the FSM matrix introduced in Section 3 can be derived. Each element FSM is equal to zero when leakage $j$ does not affect pressure in node $i$ and it is equal to 1 when leakage $j$ affects node $i$. The aim is to generate the signature matrix from the normalised sensitivity matrix. In Figure 3, it can be seen that all leakages affect all pressures.

A process inspired in the $\varepsilon$-method proposed by Sezer and Siljak (1986) is proposed with the aim of identifying the strongest relations between leaks and pressure measurements. In this process it is absolutely essential to choose conveniently the threshold that controls if a leak has or not an effect on a given pressure. The process proceeds as follows: those leaks that have an effect less than the given threshold are considered as a ' 0 ' in the leak signature matrix (5). Otherwise, their effect is 
considered as a ' 1 '. In this way, the sensitivity matrix is binarised based on selected threshold. Normalisation allows using a unique threshold for all sensors but the choice of the threshold is most relevant in the process. For small thresholds, all binarised matrix elements are 1 and only detection is possible. As the threshold increases more 0’s appear. When threshold approaches 1 then only diagonal is 1 and localisation is perfect (or almost perfect, simulation precision makes some nodes equally sensible to some leakages) but all sensors are needed. Figure 4 shows how the number of ones decrease as threshold approaches 1. Number of signatures increase but the significance of each sensors decays. Figure 5 shows the evolution of the number of signatures present in the matrix and the maximum number of leakages with the same signature. It corresponds to the 1613 nodes of the network in Figure 1 . Theoretically with 11 sensors (rows) there may be 2047 ( $2^{11}-1$; signature with all 0 is discarded as detection is imposed) different signatures for leakages (columns). In order to get maximum number of signatures a necessary condition is to have in each column $2^{n-1} 1$ 's, where $n$ is the number of sensors (rows). This necessary condition is fulfilled for the threshold where both lines in Figure 5 cross $(\sim 0.1)$. This is the threshold used.
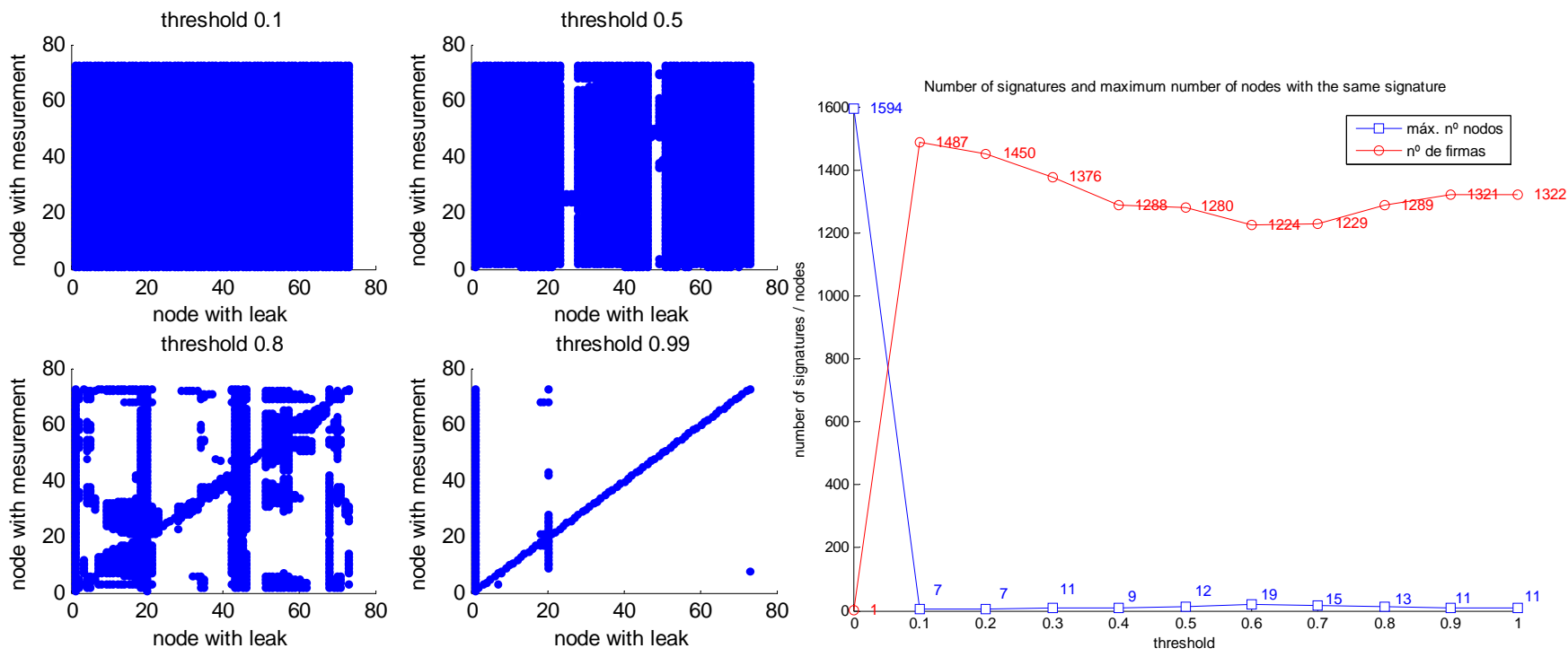

Fig. 4: Number of 1 's and 0 's depending on threshold

Fig. 5: Evolution of the signature matrix depending on threshold 


\section{SENSOR PLACEMENT ALGORITHM}

An optimal sensor placement is defined as a sensor configuration that achieves the minimum economical cost (number of sensors) while observing pre-specified performance criteria (groups of nodes that are not isolable with a minimum number of elements).

A model of water network can be represented as a graph $G=(V, E)$, where $E$ is the set of edges that represent the pipes and $V$ is the set of vertices (nodes) where pipes meet. Vertices can represent sources, such as reservoirs or tanks, where water is introduced or sinks (demand points) where water is consumed. Each pipe connects two vertices $v_{i}$ and $v_{j}$ and usually is denoted as $\left(v_{i}, v_{j}\right)$.

Using the graph representation, the problem of optimal sensor placement can be formulated as an integer programming problem, where each decision variable $x_{i}$ associated to a node $v_{i}$ of the network can be 1 or 0 , meaning respectively that the sensor will be or not installed in this node (Bagajewicz, 2000). The starting point of the algorithm is the leakage sensitivity matrix obtained by simulation binarised using the process described in Section 4. Every row corresponds to a hypothetical position of a sensor in a node while every column corresponds to a possible leak in a node. Thus, if a given element of this binary matrix contains a " 1 " means that installing the sensor in the node corresponding to this row will be able to detect the fault associated to column of this element, assuming a single leakage at time. A particular distribution of sensors (solution) is achieved by instantiating the value of decision variables $X_{i}$ to " 1 " (meaning installing the sensor) or to "0" (meaning non installing the sensor). For any particular distribution, a set of groups of indiscernible leaks appear, each group with $n_{i}$ leaks. The objective of the sensor placement algorithm is to find the sensor distribution that minimizes the number of elements for the largest set of leaks with the same signature. The objective (cost) function is therefore:

$$
J=\min _{x_{1}, \cdots, x_{n}} \max \left\{n_{1}, \cdots, n_{n_{f}}\right\}
$$

where $x_{1}, \cdots, x_{n}$ are the decision variables that determines a particular sensor distribution and $n_{i}$ is the number of nodes in group $i$ of indiscernible nodes for a given leakage $f_{i}$. In order to increase isolability this cost should be minimized but at the same time keeping the economical cost reasonable, that is installing the less number of sensor that is possible. The problem is solved for a number of sensors; this number is increased till the cost does not decrease subtantialy. A constraint is included such that all leaks should be detected. It is introduced by forcing that signature with all 0 's is not accepted. 
This optimization problem can be solved using either deterministic method based for example in Branch and Bound or heuristic methods based for example in Genetic Algorithms. The first type of methods guarantee the optimal solution but the computation time tends to be exponential with the number of nodes/faults (Sarrate et al., 2007). On the other hand, the second type of methods just guarantees a suboptimal solution that tends to the optimal one when the size of considered population tends to infinity. Besides the formulation of solutions in series of 1's and 0's are most convenient for a GA.

In Figure 6, the evolution of cost function is presented. The cost has been taken as the number of nodes in the biggest group of possible leakage isolated with a number of sensors and a threshold between 0.1 and 0.4 . A sharp improvement appears with the first sensors but adding more than 7 or 8 sensors introduce little improvement for any threshold. Therefore only 8 sensors are used.

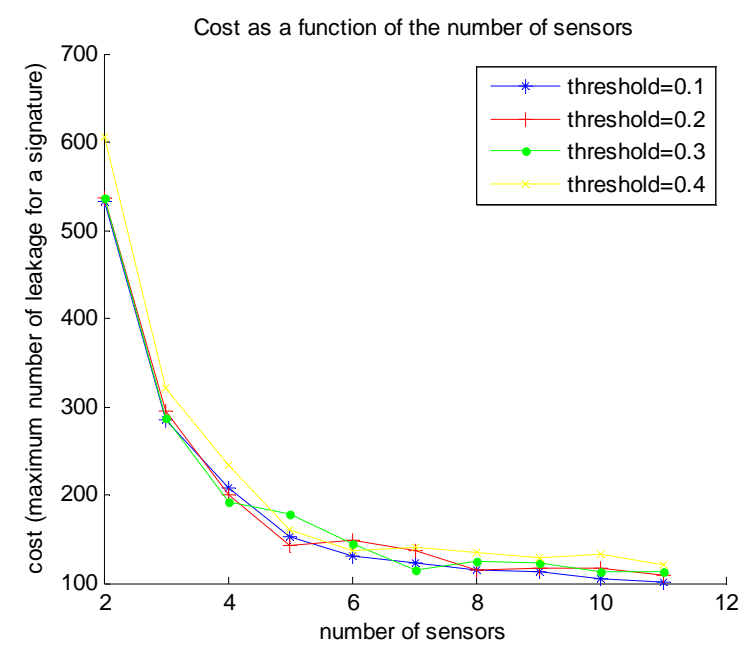

Fig. 6: Evolution of the cost function depending on number of sensors and threshold

In Figure 7, the different groups of nodes with the same leakage signature are shown. There are 39 groups and the hugest contains 190 nodes. The localization of the sensors after the optimization process is in the last figure. 

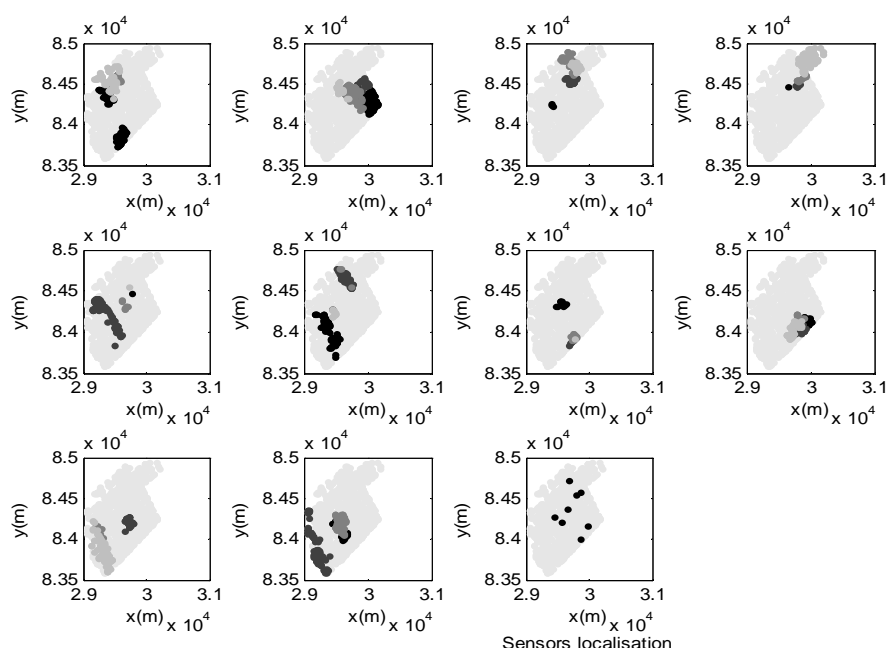

Fig. 7: Groups of nodes with the same leakage signature with 8 sensors and placement of sensors

In an ideal situation with a well calibrated network model, a leakage should be searched in one of these regions instead of the whole sector. It is important to note that regions are connected and geographically coherent. Such coherence is a major issue for further search in situ. For further details see (Pérez, 2009b).

\section{LEAKAGE DETECTION AND LOCALISATION ALGORITHM}

The localisation of leakages is based on the isolation techniques presented in Section 2. The binarised sensitivity matrix is used as a signature matrix for all leakages. In Section 3, the signatures were used to obtain an optimal distribution of the sensors and in Figure 7 the groups of nodes that generate the same signature are presented.

In the process of leak localization, the signature generated in case of having 8 sensors installed with a fault is compared with the binarised signature matrix. If the model were perfect, the leak should be localized with one measurement. Because of modelling uncertainty, the test has been done during 15 days of simulation (only the lowest consume hour is used each day when uncertainty in demands is minimal) and then three options are used to assign the observed leakage signature to a group:

- $\quad$ Mean of the sensitivities

- Mean of binarised sensitivities

- $\quad$ The voting (all days the leak is assigned to a group and that with more assignation is the elected) 
Results, even without uncertainty, were not good using any of the three decision criteria. It was due to the changing boundary conditions (pressures and flows) that affected very much the sensitivity matrixIt is necessary to generate ad hoc for each day with proper boundary conditions that are known. When a new signature matrix for each day is generated the two first approaches are useless because signature change for each iteration and means are meaningless. The third one is tested. It provided perfect results without uncertainty, $100 \%$ localisation. It means that each day the group that was signalled suitable to have a leakage contained the node with leakage. These groups were all different each instant and signature matrix is adapted to boundary conditions thus only the voting method had sense.. Thus, there are different probabilities of having a leak in a node. This appears on Table 1. It shows the number of nodes with that have been signalled 0-15 times (each for each day). The shadowed line cell corresponds to the one that contains the node with leakage. In this case, it corresponds always to the 15 . It has been done for the 39 groups (one leakage for each) that appeared in the first day (Figure 7). In Figure 8, the nodes are presented in grey scale representing the times that have been signalled to be suitable of containing a leakage. The one that contained it appears in the black area.

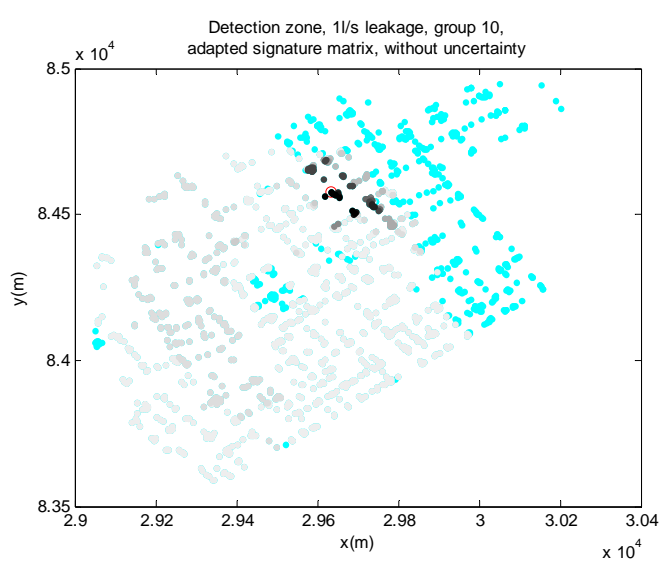

Fig. 8: Localisation of a leak in the correct zone with adapting signature matrix

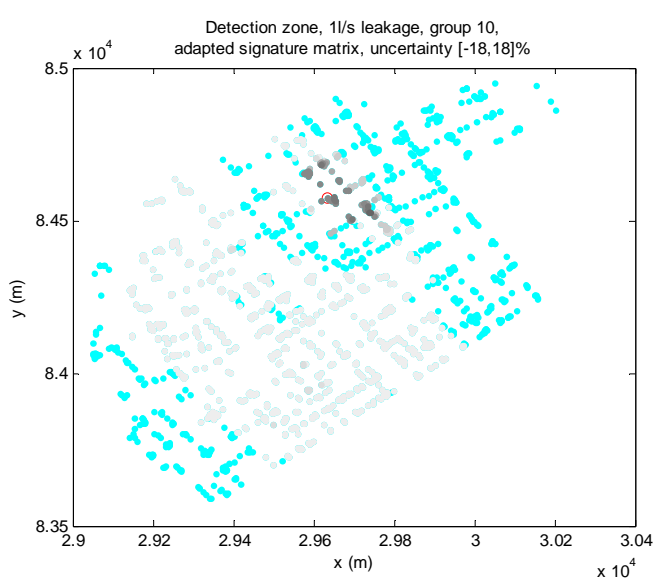

Fig. 9: Localisation of a leak in the correct zone with $18 \%$ uncertainty in the demand.

In order to test the methodology under uncertain parameters in the model, uncertainty in demands was introduced. Uncertainty was estimated using the monthly variation for a demand. It was of $18 \%$ of the total demand. Uncertainty was introduced as a coefficient multiplied to the demand of each node generated as a random number between 0.8 and 1.2. The global demand has been kept equal because it is a measured variable and affects greatly the sensitivity.

Results are presented in Table 2 and Figure 9. In this case, the leaky node is not always exactly in the most signalled group and the dark grey in the figure does not correspond to 15 but to 13 days. In Figure 9, the gray scale is lighter than in Figure 8 because there are less correct detections due to the uncertainty. 
Table 2: Results using voting criteria with uncertainty $18 \%$

Table 1: Results using voting criteria adapting signature matrix

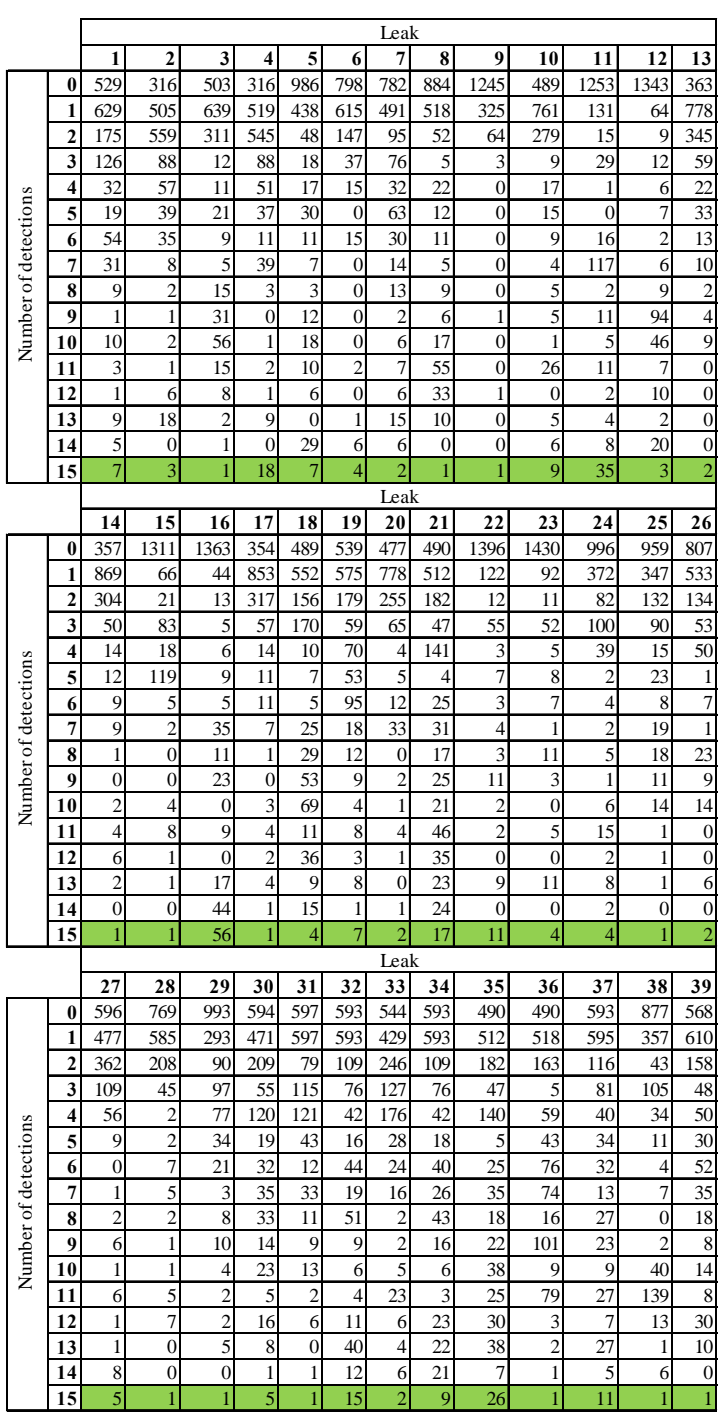

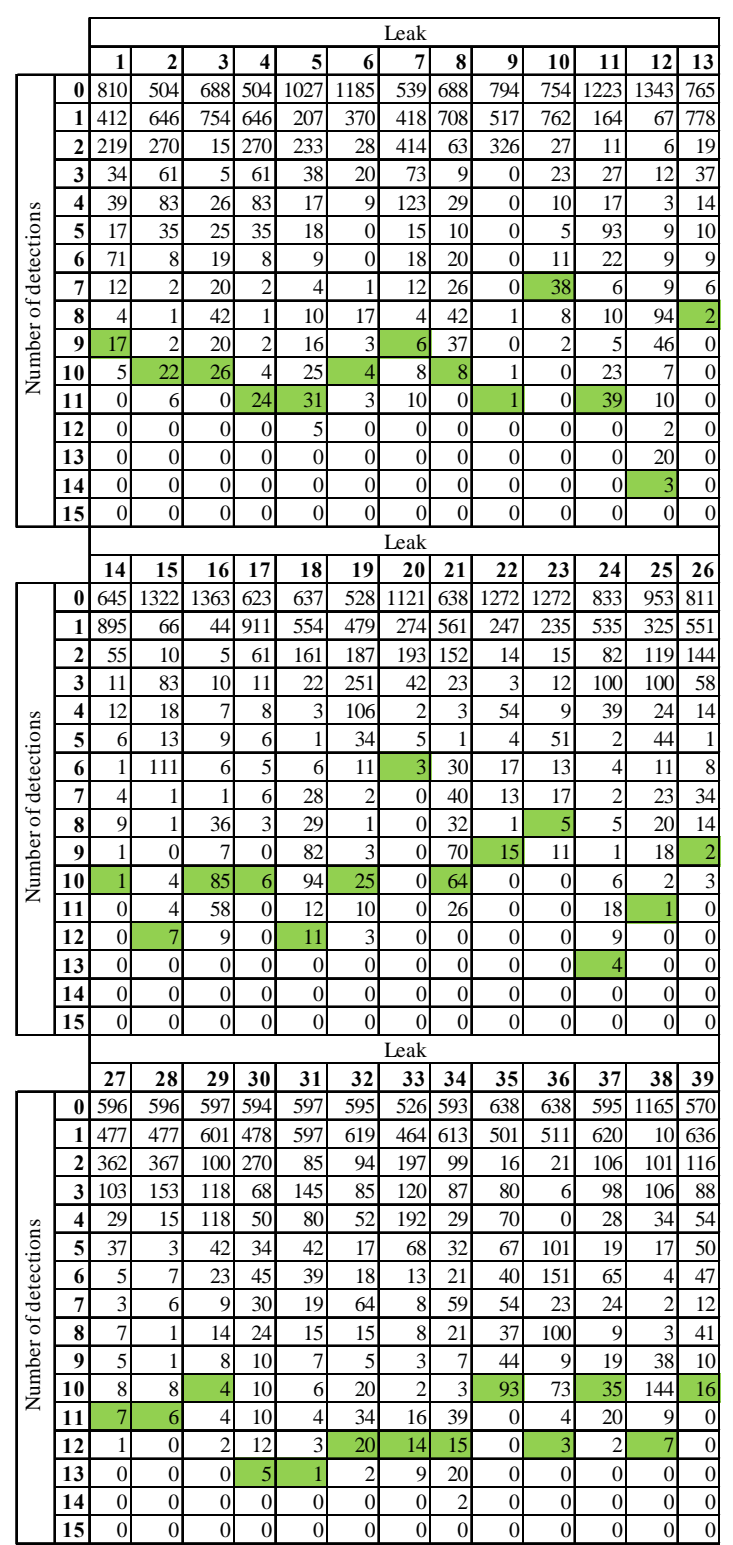

Increasing uncertainty interval, the proposed localization methodology produces poorer results. For a 50\% uncertainty leaks were not well localized but they were localized in a neighbour zone.

The main handicap of the methodology is that in a highly looped network pressure drops due to a leak are not very significant.

Therefore it demands high accuracy in transducers. Table 3 show the maximum and minimum pressure drop for leaks $0.5-10$ 1/s. In high demand hour the difference is higher but the uncertainty in demand is higher too. Thus the high cost of sensors may not guarantee good results because of uncertainties in demands. 
Table 3: Maximum and minimum pressure drop

\begin{tabular}{|c|c|c|c|c|}
\hline \multirow[t]{2}{*}{ Leakage flow [1/s] } & \multicolumn{2}{|c|}{ Minimum demand hour } & \multicolumn{2}{|c|}{ Maximum demand hour } \\
\hline & Minimal $\Delta \mathbf{P}[\mathbf{m}]$ & Maximal $\Delta \mathbf{P}[\mathrm{m}]$ & Minimal $\Delta \mathbf{P}[\mathrm{m}]$ & Maximal $\Delta P[m]$ \\
\hline 0.5 & 0.01 & 0.02 & 0.01 & 0.03 \\
\hline 1 & 0.01 & 0.04 & 0.01 & 0.06 \\
\hline 2 & 0.01 & 0.09 & 0.01 & 0.12 \\
\hline 3 & 0.01 & 0.14 & 0.01 & 0.18 \\
\hline 4 & 0.01 & 0.19 & 0.01 & 0.24 \\
\hline 5 & 0.01 & 0.24 & 0.01 & 0.31 \\
\hline 6 & 0.01 & 0.29 & 0.01 & 0.38 \\
\hline 8 & 0.01 & 0.37 & 0.01 & 0.52 \\
\hline 10 & 0.01 & 0.44 & 0.01 & 0.67 \\
\hline
\end{tabular}

\section{REAL TEST}

Results from simulation test showed that high accuracy sensors are required. Such sensors exist but represent a major investment. Before such investment is authorised real test with existing sensors were carried on. Few sensors with non optimal distribution are available. Measures have not been taken in best conditions (lower demand time). Nevertheless these results were interesting for the company in order to take further decisions and are presented in this section. Two scenarios have been used. One was a leakage forced in Enamorats DMA and the other one was a real episode of leakage in Santa Eulalia DMA.

\subsection{Enamorats DMA scenario}

Enamorats DMA model contains 260 nodes and two water input points, where a flow meter and a pressure meter are installed. Input flows in the network and pressures at these points are fixed in the simulation model, boundary conditions. In addition to this information, this DMA have 3 installed pressure sensors, which have been used to apply leakage localisation methodology. The water company provided boundary conditions (pressure and flow) and pressure inside the DMA (three sensors) data with 10 minute time-step. This information was for 5 days in the last day a leakage was forced. Table 4 shows information about this leakage.

Table 4: Leakage information in Enamorats DMA 


\begin{tabular}{lcccc} 
Flow $\left[\mathrm{m}^{3} / \mathrm{h}\right]$ & Flow $[\mathrm{l} / \mathrm{s}]$ & Leak location & Start time & End time \\
\hline 18 & 5 & Lepant/Aragó & $10: 20$ & $10: 35$ \\
14 & 3.9 & Lepant/Aragó & $10: 37$ & $10: 52$ \\
9 & 2.5 & Lepant/Aragó & $10: 53$ & $11: 08$ \\
6 & 1.7 & Lepant/Aragó & $11: 10$ & $11: 25$ \\
16 & 4.4 & Aragó 79 & $11: 53$ & $12: 08$
\end{tabular}

The first step is to verify that the hydraulic model provided is correctly calibrated. A four days simulation without any leakage has been done taking pressure values in three internal pressure sensors. The result is the pressure evolution during each day in internal pressure sensors. Differences between the model and reality are important because of demand uncertainty. The worst consequence of these results is that pressure difference caused by a leak can be hidden. To solve this problem model is corrected with the mean error during no leakage days. Real corrected pressure using these mean errors in each sensor is shown in Figure 10, compared with the simulation ones.
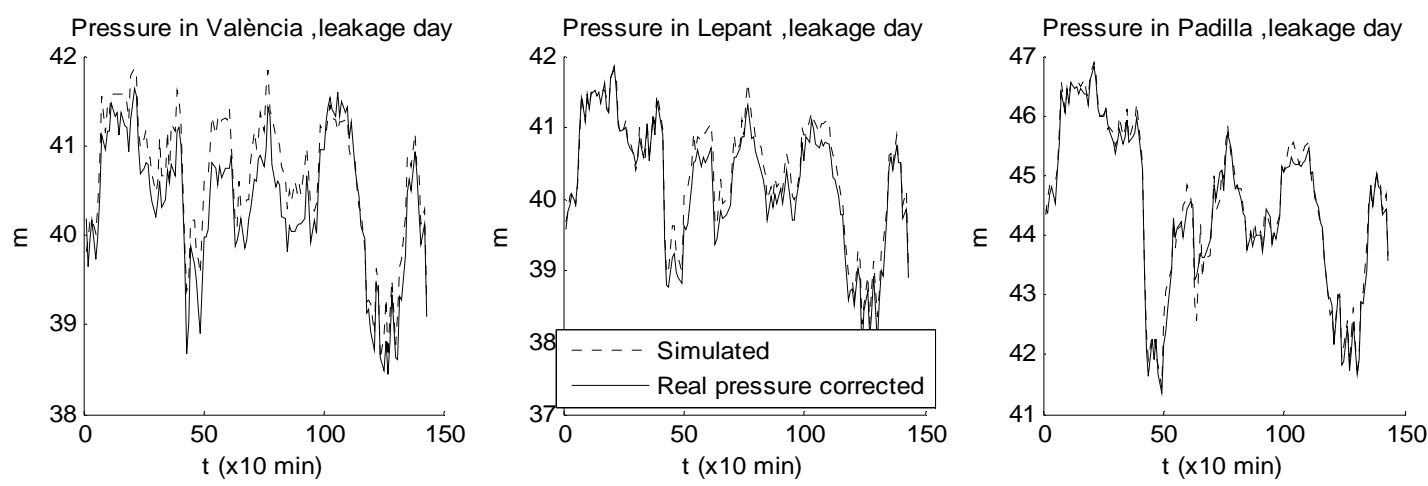

Fig. 10: Corrected real pressures compared with simulated ones.

Although a correction to real pressure has been applied no difference in the period of leakage can be observed. Thus localisation methodology is applied to see if it is possible to show more information not seen in previous figures. Leakage period duration is about one hour. For leakage period, five second step time data is given by the Water Company, but only pressures, not flows. If a ten minute step time data is used, in an hour period only 6 samples can be taken. To increase the number of samples a minute step time is proposed. To calibrate the model pressures at the input points are calculated by the mean of the last 30 second data (6 samples) and input flow is taken as a constant during 10 minutes.

To find discriminable zones obtained with installed sensors, a leak is moved for all 260 possible nodes using the model. For the leakage period two simulations are done: the first one without any leakage and the second one with a leakage moved for 260 
nodes. Forced leakage flow is not constant, as is seen in Table 3, but only ten minutes data is given for each case. For this it is assumed that the leakage flow is one of them for the whole period, $5 \mathrm{l} / \mathrm{s}$. This assumption can be justified for the fact that in a real situation the leakage flow may be variable and unknown.

In the same way as in simulation tests, leakage methodology has been applied during more than one step time. In next figures some results are shown. The first case corresponds to the leakage period. As well as in simulation, signatures matrix has been calculated depending on boundary conditions. Due to the little quantity of data, the test has been done during the whole leakage period, taking a pressure measure every minute. Results for a 0.4 threshold are shown in Figure 11 . Although some leakages are not detectable (55 nodes zone), the real leakage is outside this zone. Sensors are not located optimally, so these undetectable leakages were expected. The number of discriminable zones is four, including the non-detectable one. Leakage zone corresponds to the third group, which contains 88 nodes.

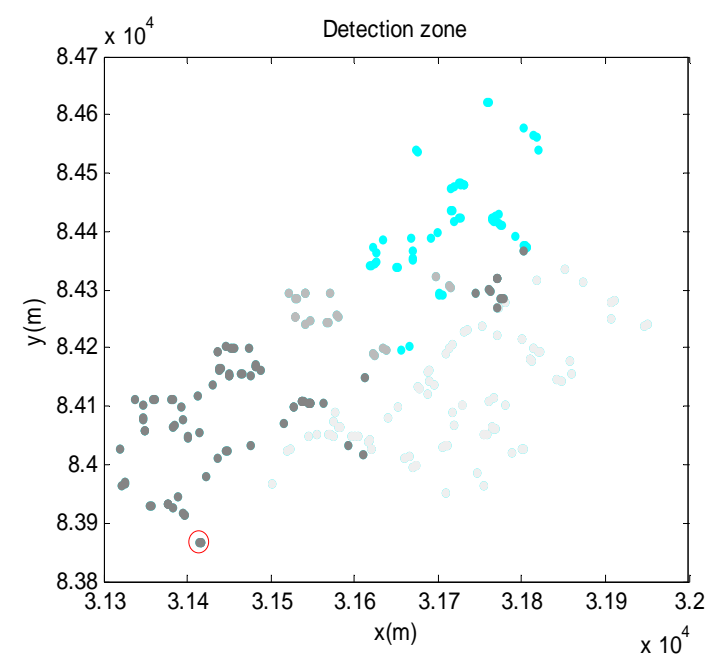

\begin{tabular}{|c|c|c|c|c|c|}
\hline \multicolumn{6}{|c|}{ Threshold $=0.4$} \\
\hline \multicolumn{4}{|c|}{ Signature } & \multirow{2}{*}{ Number of nodes } & \multirow{2}{*}{ Number of detections } \\
\hline València & Lepant & & & & \\
\hline & $\mathbf{0}$ & $\mathbf{0}$ & 0 & 5 & 0 \\
\hline & 1 & $\mathbf{0}$ & $\mathbf{0}$ & 23 & 17 \\
\hline & 1 & 1 & 0 & $8 \varepsilon$ & 31 \\
\hline & 1 & 1 & 1 & 9 & 4 \\
\hline & & & & otal $(\max =64)$ & 52 \\
\hline
\end{tabular}

Fig. 11: Leakage localisation results with a threshold of 0.4 in a leakage period.

The leakage is given in the circled node: 31 of 64 detections signalled the correct leakage zone. After this test a non leakage period is chosen to apply the methodology. At night discrepancies between reality and the model are smaller than during the day, so it is the best time to do the test. Although an important zone is signalled as a possible leakage zone, number of detections is only 9 on 42. These results are shown in Figure 12. 


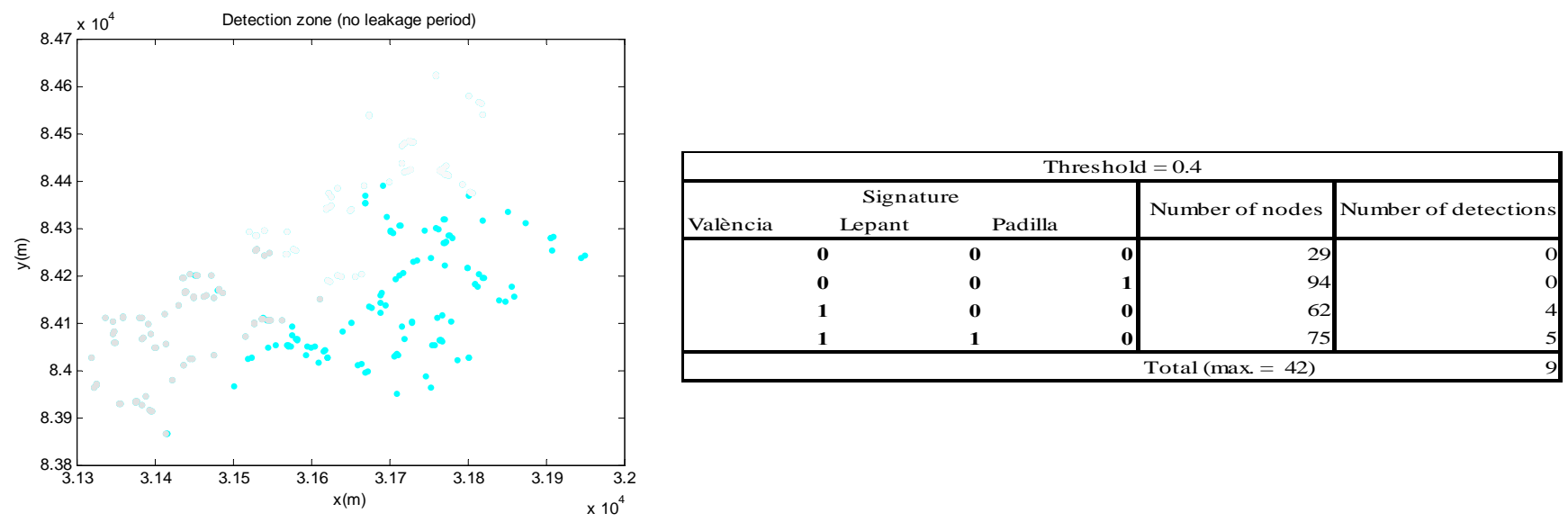

Fig. 12: Leakage localisation results with a threshold of 0.4 in a non leakage period.

\subsection{Sta. Eulalia DMA scenario}

Sta. Eulalia DMA contains 2132 nodes and four input points. In this case the number of installed sensors is four. The information provided for Sta. Eulalia DMA was for 14 days where two real leakage periods of 14 and 25 l/s mean flow. The first one was in the $15^{\text {th }}$ of September 2009 and the second one in the $9^{\text {th }}$ of September 2009 . The same methodology has been applied. Leakage periods data are shown in Table 5. The number of pressure samples to apply the localisation technique is really low, 6 in the first episode and 2 in the second one.

Table 5: Leakage information in Sta. Eulalia DMA

\begin{tabular}{cccc} 
Day & Start time & End time & Mean flow \\
\hline $09 / 09 / 2009$ & $16: 35$ & $17: 30$ & $25 \mathrm{l} / \mathrm{s}$ \\
$15 / 09 / 2009$ & $12: 15$ & $12: 30$ & $14 \mathrm{l} / \mathrm{s}$
\end{tabular}

Detection results for the first and the second episodes are shown in Figure 13. Although the leakage is inside the detection zone in both cases (in the circled zones), it is not situated in the most probable zone. 

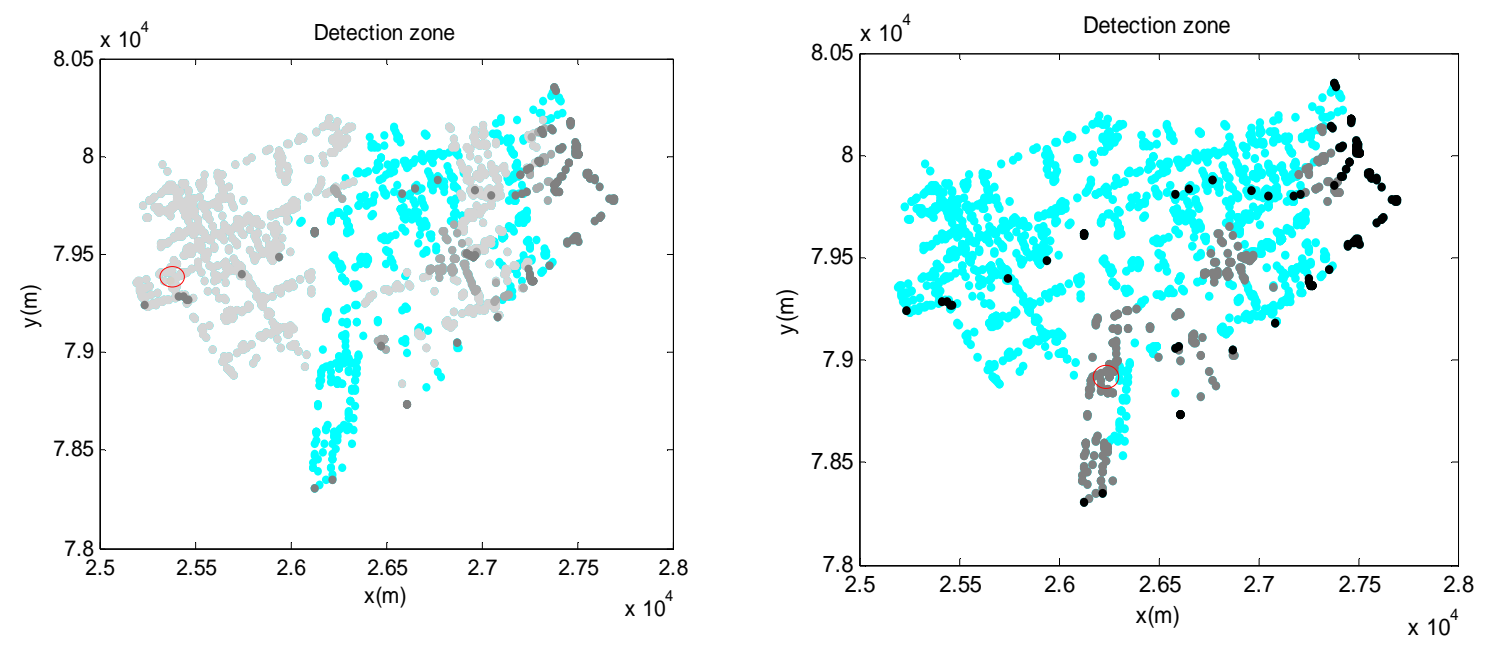

Fig. 13: Leakage localisation results for the first and the second leakage period in Sta. Eulalia

\section{CONCLUSIONS}

A leakage localization method based on the pressure measurements and sensitivity analysis of nodes in a network has been proposed. The leakage localization methodology is founded in standard model based fault diagnosis well established theory.

In order to maximise the isolability with a reasonable number of sensors an optimal sensor placement methodology based on genetic algorithms is also proposed. The objective function in the minimisation process was the size of the maximum group discriminated.

To assess the validity of the proposed approach, it has been applied to real DMA of Barcelona network in real and simulated leak scenarios. Models and information were provided by the water company. For these sectors (DMA), the sensor placement and the leakage detection and localization methodologies have been applied with successful results even in presence of demand uncertainty.

An issue in the process is to recalculate the sensitivity matrix for each boundary condition using the simulation model because of the high dependence of it to global consumption. This approach is being currently developed using linear parameter varying (LPV) models that consider the consumption as a scheduling variable (Vento, 2009). 


\section{ACKNOWLEDGMENT}

The authors wish to thank the support received by WATMAN ref. DPI2009-13744 of the Spanish Ministry of Education and

PROFURED project funded by AGBAR-CETAQUA.

\section{REFERENCES}

Bagajewicz, M., Design and Upgrade of Process Plant Instrumentation. Lancaster, PA: Technomic Publishers. 2000.

Blanke, M., Kinnaert, M., Lunze, J., Staroswiecki, M. (2006). Diagnosis and Fault-tolerant Control, $2^{\circ}$ Edition, Springer.

Brdys, M. A., Ulanicki, B. (1994). Operational control of water systems: Structures, algorithms and applications. Prentice Hall International, UK.

Chen, J., Patton, R.J. (1999) Robust Model-Based Fault Diagnosis for Dynamic Systems. Kluwer Academic Publishers.

Colombo, A.F., Lee, P., Karney, B.W. (2009) “A selective literature review of transient-based leak detection methods”. Journal of Hydro-environment Research, 2, pp. 212-227.

Farley, M., S. Trow (2003). Losses in Water Distribution Networks. IWA Publishing UK, 2003.

Gertler, J.J. (1998), Fault Detection and Diagnosis in Engineering Systems, Marcel Dekker, 1998.

Perez, R., S. de las Heras, J. Aguilar, J. Pascual. A. Peralta (2009a), “District management areas characterisation in water network based on clustering”. Water Science Technology, Vol 9 No 5 pp 591-600, 2009.

Pérez, R., Puig, V., Pascual, J., Peralta, A., Landeros, E. and Jordanas, Ll.. (2009b) “Pressure sensor distribution for leak detection in Barcelona water distribution network”. Water Science \& Technology, Vol 9 No 6 pp 715-721.

Pudar, R.S., Ligget, J.A. (1992) Leaks in Pipe Networks. Journal of Hydraulic Engineering. Vol. 118, No. 7, July 1992, pp. 1031-1046. 1992.

Lambert, A. (1994), Accounting for losses: the Bursa and background concept. (BABE)IWEM Journal, April 1994, 8(2), 20514.

MacDonald G. (2005) “DMA Design and Implementation”, a North American Context. Leakage Conference, IWA. 
Ragot, J., Maquin, D. (2006). “Fault measurement detection in an urban water supply network”. Journal of Process Control, Volume 16, Issue 9, Pages 887-902.

Sarrate, R., Puig, V., Escobet, T., Rosich, A. (2007). Optimal Sensor Placement for Model-based Fault Detection and Isolation. 46th IEEE Conference on Decision and Control. New Orleans, USA.

Sezer, M. E. and Siljak, V. Nested epsilon-decomposition and clustering of complex systems. Automatica, 22(3):321- 331, 1986.

Vento, J., Puig, V. (2009) Leak Detection and Isolation in Pressurized Water Networks Using Interval LPV Models. $7^{\text {th }}$ Workshop on Advanced Control and Diagnosis (ACD’09). Zielona-Gora, Poland. 